\title{
Experience gained during Manufacture and Test of the W7-X Superconducting Magnets
}

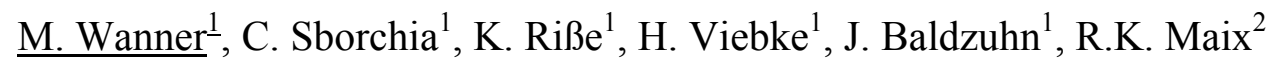 \\ 1) Max-Planck-Institut für Plasmaphysik, EURATOM Association, \\ D-17491 Greifswald, Germany \\ 2) Atomic Institute, University of Technology, EURATOM Association, \\ A-1020 Vienna, Austria
}

Corresponding author: Tel.: +49-3834-882701; fax: +49-3834-882709; E-mail address: manfred.wanner@ipp.mpg.de

\begin{abstract}
The optimised stellarator WENDELSTEIN 7-X (W7-X) is presently being assembled at the Greifswald branch of IPP. The specific field configuration of W7-X is realised by a periodic arrangement of 50 non-planar and 20 planar superconducting coils in five modules. The winding packs are wound from a cable-in-conduit conductor and embedded in massive steel casings. Tests during production and at operational conditions are performed to verify that essential requirements are met. Manufacture of the non-planar and planar magnets is in an advanced stage. The manufacturer had to solve several problems and consider major design changes during advanced stages of manufacture. Meanwhile, several coils have been delivered and tested and assembly of the first half-module has started. The paper gives an overview of the production status and highlights the lessons learned during fabrication and test of the coils.
\end{abstract}

Keywords: WENDELSTEIN7-X, superconducting magnets

\section{Introduction}

W7-X is a superconducting fusion device which shall allow steady state operation and prove the favourable operational properties of the optimised stellarator principle [1]. The magnet 
system of W7-X is composed of five identical modules which are divided into two flipsymmetric half-modules. In each half-module five differently shaped non-planar coils and two slightly different planar coils are bolted to a massive steel structure. Superconducting bus bars link all coils and connect the coils with the current leads. The overall properties of the magnet system are summarised in table 1 .

The standard configuration is characterised by a magnetic induction of $2.5 \mathrm{~T}$ at the plasma axis and a rotational transform $\mathrm{l}_{\mathrm{a}}=5 / 5$ at the plasma boundary. By varying the currents in the non-planar and planar coils the field on the axis can be increased to $3 \mathrm{~T}$ and $\mathrm{l}_{\mathrm{a}}$ can be varied between 5/6 and 5/4 [2]. Magnetic islands which are formed under resonant conditions at the plasma boundary are used to control particle and energy exhaust in an open divertor.

To achieve the physics goals the errors of the magnetic field $\Delta \mathrm{B} / \mathrm{B}$ need to be kept below $10^{-4}$. This means that the individual shapes of the ten winding packs of one type must be reproduced with an accuracy of a few millimetres.

To sustain the large electromagnetic forces the winding packs of the coils are stiffened by massive steel casings. Each coil is rigidly bolted at two extensions to a massive coil support structure and connected to the neighbouring coils by different types of inter-coil supports [3]. Achieving the required accuracy requires considerable effort during manufacturing, surveying and controlling the interfaces and all steps of assembly.

\section{Superconductor}

All coils use a cable-in-conduit conductor which is composed of $243 \mathrm{NbTi}$ strands wound to a cable and enclosed by an aluminium jacket. The void space in the conductor amounts to $37 \%$ and is used for the helium coolant. Meanwhile the consortium EAS/OCSI (formerly VAC/EM) has completed delivery of all 360 required conductors with typical lengths between 120 and $180 \mathrm{~m}$ and some additional lengths for the bus system and as spares. 
Strand production was shared between the EAS and OCSI. Critical currents $I_{c}$ from both suppliers were above the specified $150 \mathrm{~A}$ at a temperature of $4.2 \mathrm{~K}$ and a magnetic field of $6 \mathrm{~T}$. The strand surface was specified as naturally oxidized. Actual surface colours ranged from metallic blank to dark blue. Tests at Paul Scherrer Institute (PSI) with heavily oxidized strands showed, however, that the contact resistance between the strands was not affected. The specified RRR value of 160 for the $\mathrm{Cu}$ matrix of the strands was also exceeded but showed significant scatter. Whereas the strands from OCSI were heat treated before cabling the EAS strands reached their specified RRR only after the heat treatment of the winding pack during the embedding process.

At OCSI the strands were cabled in 5 stages. Small differences of the surface roughness of the strands from different suppliers affected the cabling process significantly and required special handling. Some conductors showed broken strands. Following investigations at PSI a maximum of one broken strand every $50 \mathrm{~m}$ was accepted under the provision that these conductors were only used for the outer turns, operating at a reduced magnetic field. Jacketing of the cable was performed by Alu-Menziken under responsibility of EAS. For the jacket the Al alloy 6063 was chosen because it is soft in the co-extruded and water quenched state and can be hardened at $160{ }^{\circ} \mathrm{C}$ to reach the yield strength of $285 \mathrm{MPa}$ which is required during operation.

The flow resistance of the conductor was measured after several steps of production. The data showed large scatter which could not be explained so far. Moreover the flow resistance decreased during winding by approx. $7 \%$ indicating relocations of the strands due to bending. Acceptance of the conductor finally required to release the tolerance on the void fraction from $37 \pm 1 \%$ to $37 \pm 2 \%$ and to adopt variations of the flow rate of up to $\pm 20 \%$.

PSI also compared the operational behaviour of the W7-X conductor with the extrapolations from single strand measurements (see Fig. 1). At $6 \mathrm{~T}$ and temperatures above $5 \mathrm{~K}$ the critical 
current $I_{c}$ follows the expected trend from the strand measurements indicating that no degradation has occurred during the co-extrusion process. At temperatures below $5 \mathrm{~K}$ the current is limited at approx. $21 \mathrm{kA}$ well below the expected critical current because of premature quenches. Since W7-X will not be operate at such high currents this will not affect the coil behaviour.

\section{Non-Planar Coils}

\subsection{Winding Packs}

The non-planar coils are manufactured by the Babcock-Noell GmbH (BNG)/Ansaldo Superconduttori Consortium [4]. Winding of the coils was performed in parallel on three manufacturing lines at Ansaldo, and at two lines at the BNG sub-contractor, ABB. Meanwhile all 50 winding packs are fabricated and delivered for integration.

The accuracy and reproducibility of the geometry of the winding packs has met the specification. The average absolute deviation is lower than $3 \mathrm{~mm}$. The average relative deviation with respect to the average shapes of each coil type is $<2 \mathrm{~mm}$ [5].

After winding and application of the ground fibreglass insulation, the winding packs are vacuum pressure impregnated with epoxy resin. The six conductor lengths of each winding pack are electrically connected in series through joints with a resistance of typically $1 \mathrm{n} \Omega$. The helium pipes are electrically insulated from the electrical circuit by potential breaks. (see Fig. 2)

After fabrication some winding packs showed inter-turn shorts. Three winding packs could be repaired; one winding packs had to be rebuilt because the short was not accessible. Special skills were required to qualify the aluminium welds between the conductor jacket and the interlayer joints thereby avoiding overheating the superconductor strands.

\subsection{Casing}


The coil casings consist of two casted half rings of $316 \mathrm{LN}$ alloy and several connecting blocks. All 100 half rings are cast, heat-treated and machined. The quality of the castings is very good in terms of weldability, mechanical properties at cryogenic temperatures and maintaining geometrical tolerances.

At the beginning, defects in the castings could only be detected in regions below $70 \mathrm{~mm}$ thickness. When new defects became visible after the final machining of the casings in regions with larger thickness, $\mathrm{BNG}$ launched a high energy radiation inspection campaign using LINACs. This required repair of defects in most of the casings and especially in the regions of the reinforcing ribs and at the corners of the winding packs by local machining and TIG welding.

\subsection{Integration of the coils}

Assembly of the non-planar coils is done at the BNG production facility in Zeitz (Fig. 3). After positioning of the winding pack the two half casings are welded together with two longitudinal TIG welds. Then, the gap between winding pack and casing is filled with quartz sand and epoxy resin. During this operation the casing is heated to about $100{ }^{\circ} \mathrm{C}$, while the winding pack is kept at ambient temperature. During re-cooling to room temperature the winding pack is pre-stressed. This pre-stress is released during cool down to $4 \mathrm{~K}$ because of the differential contractions between the conductor and the steel casing. After welding of additional support blocks to the casing, the coil surface is machined to the required precision on a five-axis $\mathrm{CNC}$ machine. During a final survey the outer contour of the coil casing, the locations of the reference pins and of the coil fixtures are checked. The accuracy is within $0.5 \mathrm{~mm}$ for the reference pins and coil fixtures, $2 \mathrm{~mm}$ for the inner surface and $5 \mathrm{~mm}$ for the outer surface of the casings.

Following the final machining, the casing cooling system is mounted. Strips of highly conductive copper are welded onto the surface of the casings and soldered to stainless steel 
tubes. The usage of copper strips reduce eddy current heating of the coils in case of a rapid shut-down of the magnet system.

Already in 2003 detailed design work on the coil supports has required the reinforcement and remachining of the support blocks, to be able to connect the coils through high strength bolts with the support ring. Mid of 2004 a change of the design of the inter-coil support elements, in particular narrow support elements and lateral supports, has required again the remachining of all completed coils, including some coils which were already tested at Saclay. The rework has also required the removal of several parts of the casing cooling tubes and resoldering of the copper stripes. To avoid a new cold test of the first four coils the orbital welds on the tubes have been subjected to local thermal cycles at $77 \mathrm{~K}$ followed by a leak test. After the appearance of corrosion on the casing cooling of a planar coil (see below) it was concluded that also the soldering of the copper stripes to the steel tubes of the non-planar case cooling might exhibit pitting corrosion. Entrapped chloride residues were suspected to trigger an autocatalytic corrosion process in the presence of humid atmosphere. This risk has been

eliminated for future coils by pretinning the tubes and carefully rinsing them thereafter. On 18 finished coils the cooling pipes could not be replaced anymore. Preventive actions were taken to ensure operation of W7-X also with reduced case cooling in the unlikely event of a leak in a pipe.

The coils are finished by mounting quench detection (QD) wires, temperature sensors and strain gauges. During final work acceptance the coils undergo a pressure and flow test, an integral helium leak test, and a $13 \mathrm{kV}$ DC as well as $\pm 2 \mathrm{kV}$ AC high voltage (HV) tests of the insulation. By end of July 2006, 14 coils were delivered; the remaining 36 coils are in different phases of integration.

\subsection{Electrical Insulation and Paschen Tests}


During a rapid shut-down the coils induce voltages of up to $\pm 4 \mathrm{kV}$ which in the worst case could coincide with a degraded insulation vacuum. For this reason the coils must be insulated in a Paschen-proof manner. The ground insulation of the coils consists of $5 \mathrm{~mm}$ glass-epoxy composite impregnated under vacuum. In the termination area where the interlayer joint and terminals are assembled, the insulation is completed manually with glass tapes and wet resin cured at lower temperature. The thickness has also been reduced in this area to 2.5-3 $\mathrm{mm}$. The first high voltage tests performed at reduced pressure revealed partial discharges at voltages significantly below the specified $9.1 \mathrm{kV}$, which were due to a weak insulation of the QD cables. It was then necessary to select and qualify a new cable. The final design uses Kapton insulated wires enclosed by a metallic shield. A connector to join the new cable to the existing wires in the winding pack was manufactured and tested by IPP [6].

Due to the concern about the quality and integrity of the insulation in the terminal area, IPP requested to add HV tests at reduced pressures (Paschen tests) during the work acceptance tests of the coils. These tests are carried out up to $9.1 \mathrm{kV}$ in a vacuum between 100 and 1 mbar in the vacuum tank, which is used for the final pressure/leak test. During such Paschen tests further weak areas were detected and need to be repaired in the termination area where voids were not filled properly with glass material and/or resin. Meanwhile another vacuum tank has been installed at IPP to perform additional Paschen tests on all coils after the cold test and before assembly.

\section{Planar Coils}

The 20 planar coils are produced by Tesla Engineering [4]. All winding packs are manufactured. At the end of July 2006 three coils have been tested and delivered to IPP (see Fig. 4). Five coils are waiting for being tested at Saclay; the remaining coils are in different stages of integration. Due to the planar D-shaped geometry, the winding packs could be 
fabricated with sufficient accuracy on a turn table. The side walls and the lids of the casings are made from plate material and fixed by shear pins and bolts.

The manufacturing process has shown problems similar to the non-planar coils. This holds in particular for the Al welding of the interlayer joints, the embedding and preloading procedure of the casings, the high voltage strength of the QD cables and the quality of the insulation in the termination area. The QD cables had also to be replaced and high voltage tests in Paschen minimum conditions are being performed at Saclay and Greifswald.

IPP performs the leak tests of all winding packs before embedding as well as on the finished coils. This is being done by a leak test method with $\mathrm{SF}_{6}$ developed by FZK. Leaks are accumulated in a plastic bag around the coil and detected by laser spectroscopy. After final machining of the coils, surveys of the coil connections, the positions of the reference pins and the contour of the casings are being performed by JET with digital photogrammetry.

During the cold tests at Saclay two coils have shown cold leaks during cool-down at about 100-150 K, probably due to failures in the Al welds of the joints and were repaired after a new qualification of the welding process. A third coil leaked due to crack corrosion in a thin walled $(0.7 \mathrm{~mm})$ casing cooling tube. Corrosion was caused by residuals of chloridric acids in the solder flux, which were entrapped between the cooling pipes and the $\mathrm{Cu}$ heat sinks after soldering. The problem was solved by increasing the wall thickness of the tubes to $1.2 \mathrm{~mm}$ and by pre-tinning the pipes with a solder paste, which does not require aggressive flux.

\section{Cold Tests}

All superconducting coils are subjected to tests at cryogenic temperature at CEA in Saclay. The test facility consists of two cryostats, each one able to host two coils of any type. The test procedure comprises high voltage tests and leak tests at ambient and cryogenic temperatures, pressure drop measurements, energisation to nominal current, and a complete quench at nominal current in the self-field [7]. The quench conditions are approached in temperature 
steps of $0.1 \mathrm{~K}$. The coil is kept just below the quench temperature (safety margin test) for $30 \mathrm{~min}$. The specified current margin for the non-planar coils $(35 \mathrm{kA})$ at $4.0 \mathrm{~K}$ and $6 \mathrm{~T}$ translates to a quench temperature of $5.7 \mathrm{~K}$ at $17.6 \mathrm{kA}$ in the self-field. In all tested coils, the quench temperature was above $6 \mathrm{~K}$, which represents some additional margin. Following these results the number of quench tests was reduced to only the first two of ten coils of one type.

\section{Summary}

The manufacture of the superconducting non-planar and planar coils of W7-X is well advanced. Production of the superconductor, the casings and the winding packs of the coils is complete. 14 non-planar and 8 planar coils have been finished. During several tests the design and the quality of production was proven. Several technical problems asked for corrective actions which were solved by a close cooperation between IPP and the companies. Paschen tests showed their effectiveness and should become mandatory. Meanwhile production of the coils has reached series character. Production of the planar coils is expected to finish at the beginning of 2007. By mid of 2008 all non-planar coils shall be finished.

\section{References}

[1] J. Nührenberg et al, Trans. Fusion Technol. 27, 71 (1995)

[2] F. Wagner et al., Physics, technologies, and status of the Wendelstein 7-X device, 20th IAEA Fusion Energy Conf., Villamoura, (2004)

[3] F. Schauer, Status of Wendelstein 7-X Construction, this conference

[4] C. Sborchia et al., Progress in the design, manufacture and testing of the W7-X superconducting magnets, IEEE Transactions of Appl. Superconductivity, 16, 848 (2006) 
[5] M. Wanner, T. Andreeva, T. Bräuer, J. Kißlinger, Accuracy of the magnet configuration of WENDELSTEIN 7-X, Proc. $31^{\text {st }}$ EPS Conf. on Plasma Phys, London, ECA vol. 28G O-4.05 (2004)

[6] B. Petersen-Zarling et al., Experience with high voltage tests of the W7-X magnets in Paschen-minimum conditions, this conference

[7] H. Ehmler et al., Review of the Acceptance tests of the W7-X superconducting magnets, this conference 
Tabel 1. Basic parameters of the magnet system of W7-X

\begin{tabular}{|ll|}
\hline - major/minor plasma radius & $5.5 / 0.53 \mathrm{~m}$ \\
- number of non-planar/planar coils & $50 / 20$ \\
- rotational transform at plasma boundary & $5 / 6 \ldots .5 / 4$ \\
- cold mass & $395 \mathrm{t}$ \\
- max. magnetic field on the axis & $3 \mathrm{~T}$ \\
- max. magnetic field at the conductor & $6.7 \mathrm{~T}$ \\
- maximum current in non-planar coil & $18.2 \mathrm{kA}$ \\
- operation temperature & $3.4 \mathrm{~K}$ - $3.9 \mathrm{~K}$ \\
- magnetic energy of coils & $900 \mathrm{MJ}$ \\
\hline
\end{tabular}


Figure captions:

fig. 1 Critical current Ic of the conductor vs. temperature $\mathrm{T}$ showing premature quenches fig. 2 Terminal area of a non-planar winding pack

fig. 3 Assembly of the winding pack into the casing at BNG (Zeitz)

fig. 4 Stringing of a planar coil across the plasma vessel 


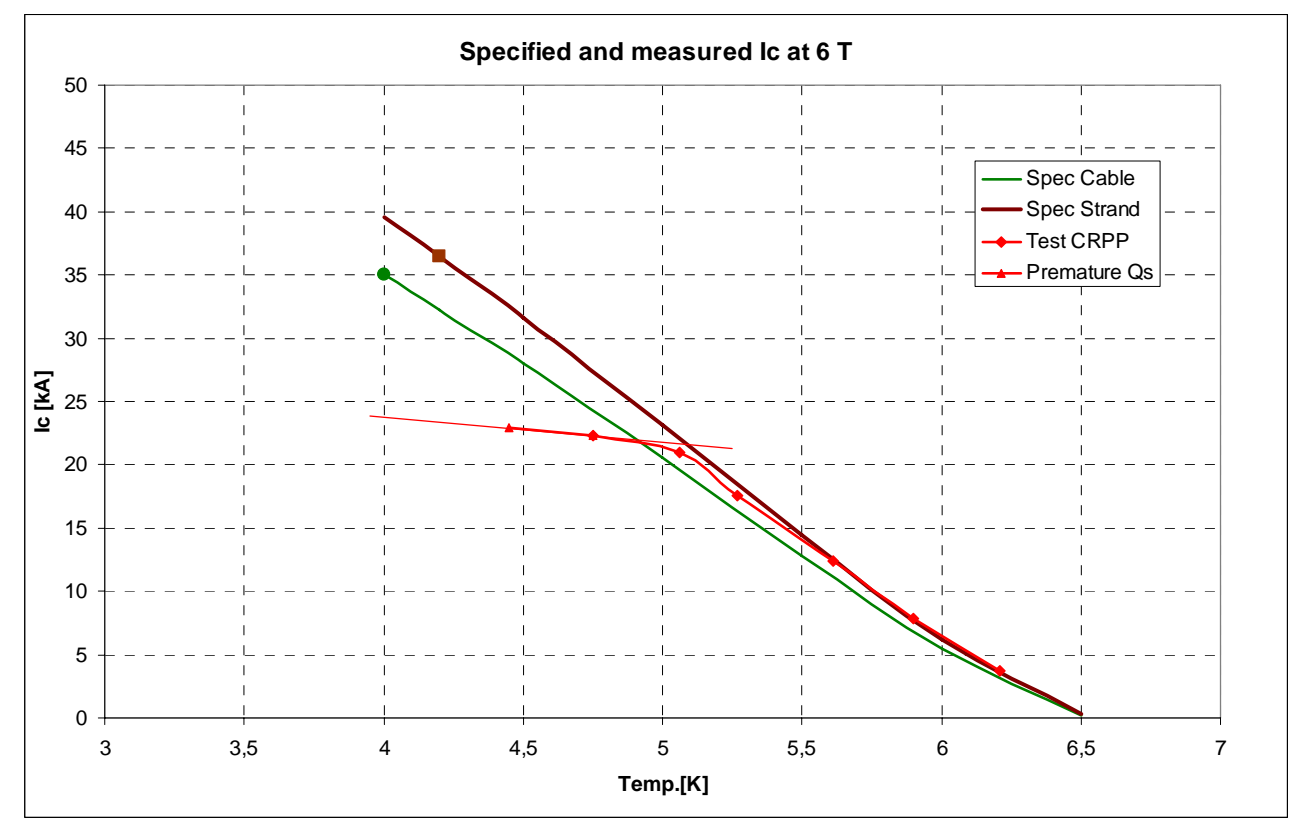

Fig 1: Critical current Ic of the conductor vs. temperature T showing premature quenches

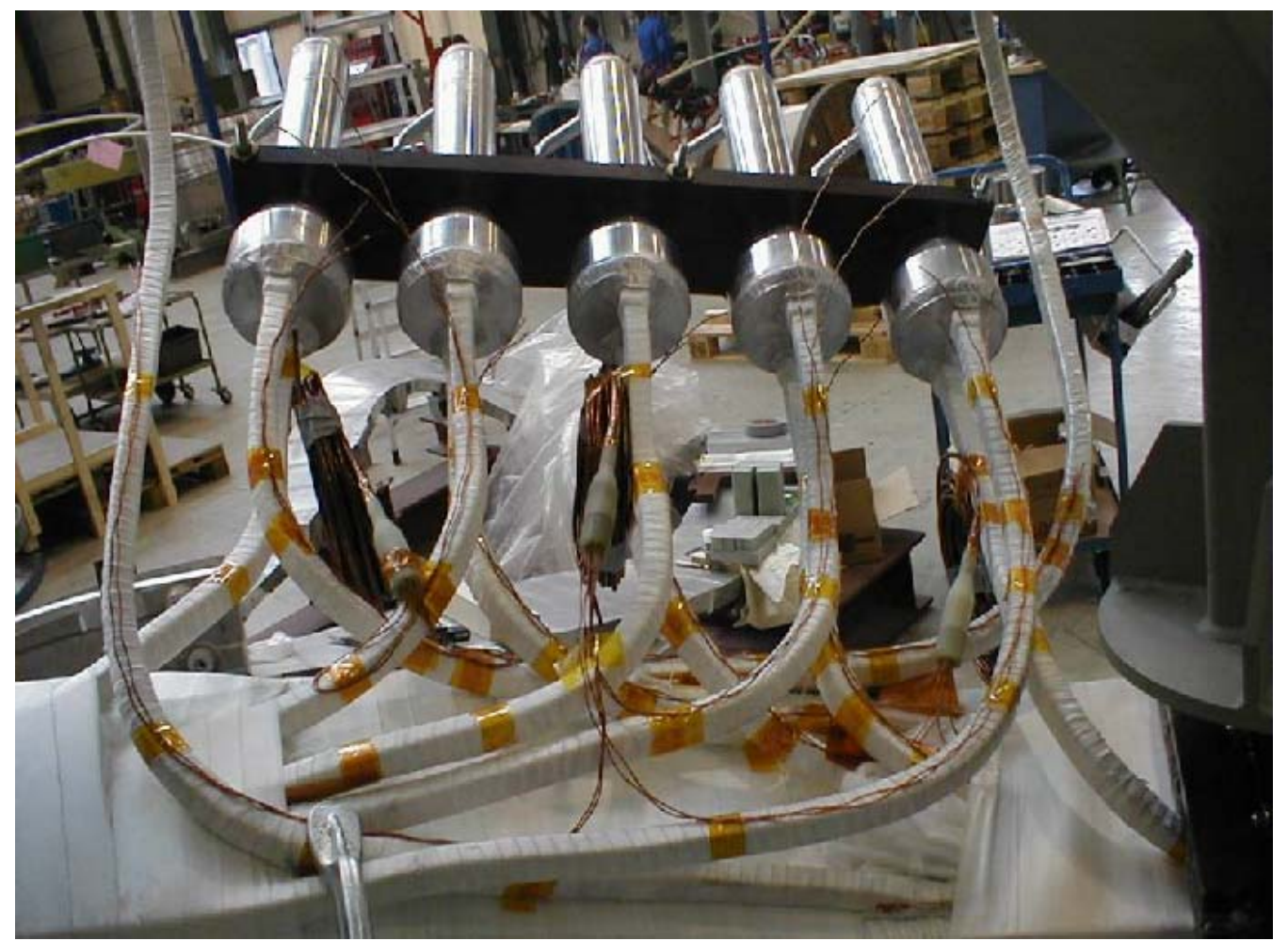

Fig 2. Terminal area of a non-planar winding pack 


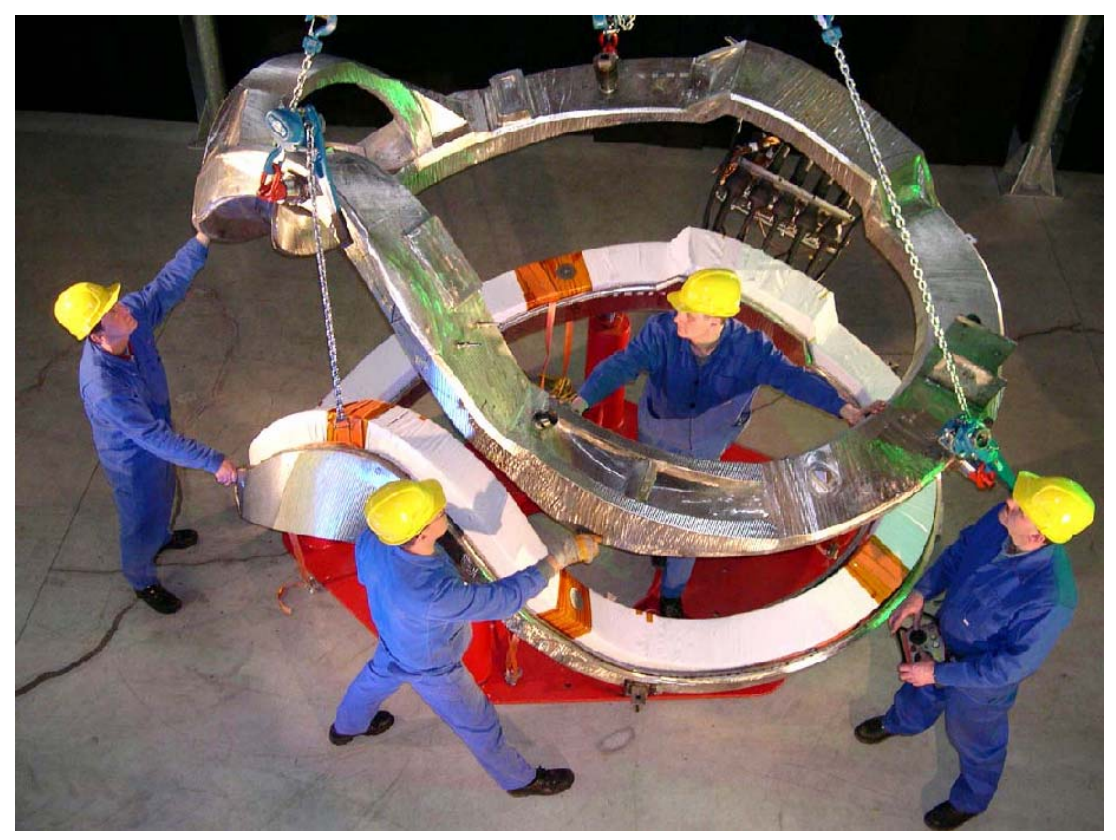

Fig 3: Assembly of the winding into the casing at BNG (Zeitz)

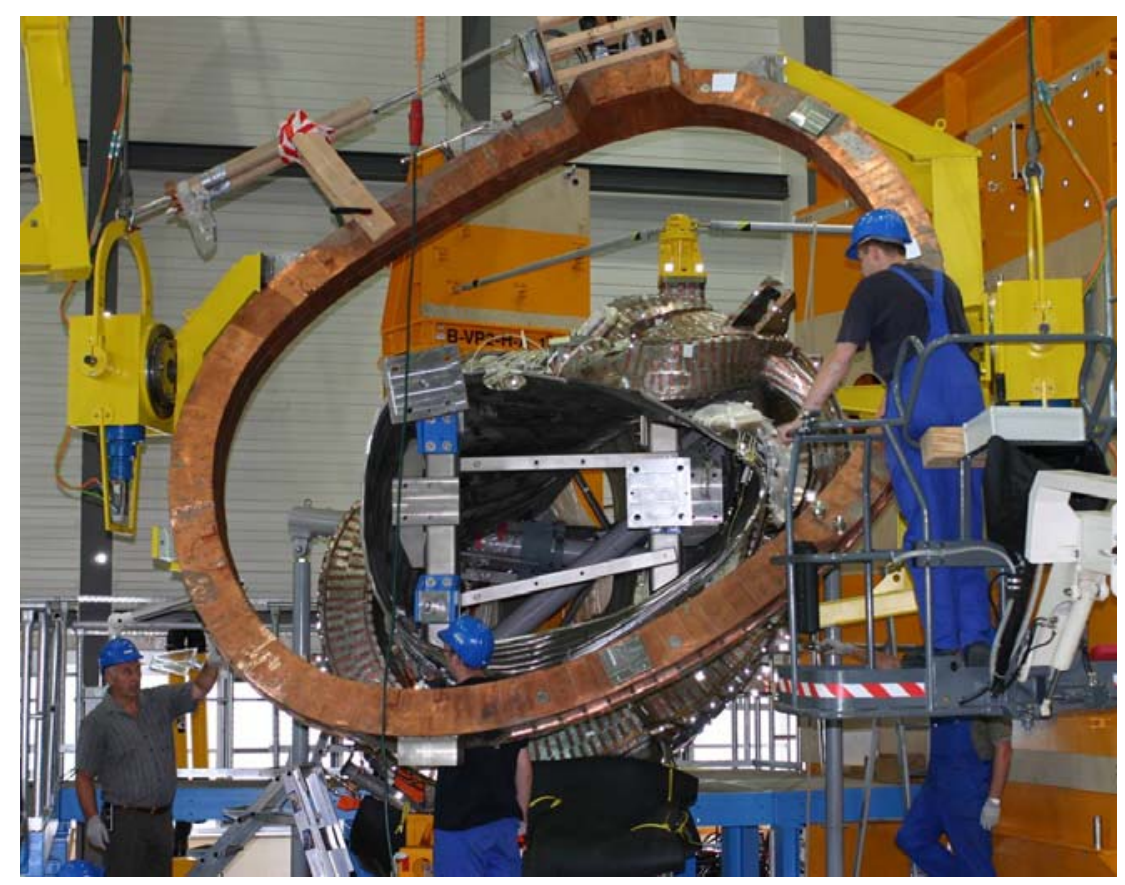

Fig. 4: Stringing of a planar coil across the plasma vessel 